\title{
On the interchannel interference in digital communication systems, its impulsive nature, and its mitigation
}

\author{
Alexei V Nikitin ${ }^{1,2}$
}

\begin{abstract}
A strong digital communication transmitter located in close physical proximity to a receiver of a weak signal can noticeably interfere with the latter even when the respective channels are tens or hundreds of megahertz apart. When time domain observations are made in the signal chain of the receiver between the first mixer and the baseband, this interference is likely to appear impulsive. Understanding the mechanism of this interference is important for its effective mitigation. In this article, we show that impulsiveness, or a high degree of peakedness, of interchannel interference in communication systems results from the non-smooth nature of any physically realizable modulation scheme for transmission of a digital (discontinuous) message. Even modulation schemes designed to be 'smooth', e.g., continuous-phase modulation, are, in fact, not smooth because their higher order time derivatives still contain discontinuities. When observed by an out-of-band receiver, the transmissions from these discontinuities may appear as strong transients with the peak power noticeably exceeding the average power, and the received signal will have a high degree of peakedness. This impulsive nature of the interference provides an opportunity to reduce its power by nonlinear filtering, thus improving quality of the receiver channel.
\end{abstract}

Keywords: electromagnetic interference, impulsive noise, interchannel interference, nonlinear differential limiters, nonlinear filtering, peakedness

\section{Introduction}

Nowadays, it is becoming more and more common that multiple digital communication devices, including wireless, coexist and concurrently operate in close physical proximity. A typical example would be a smartphone equipped with WiFi, Bluetooth, and GPS, and capable of operating at various data protocols and in multiple frequency bands. This physical proximity, combined with a wide range of possible transmit and receive powers, creates a variety of challenging interference scenarios. Multiple sources of empirical evidence indicate that such interference often manifests itself as impulsive noise $[1,2]$, which in some instances dominates over the thermal noise $[1,3]$. However, there are many unanswered questions regarding the origins and the particular manifestations of this type of noise. For example, a strong close transmitter (say, WiFi) can noticeably interfere

Correspondence: avn@avatekh.com

${ }^{1}$ Avatekh Inc., 900 Masachusetts Street, Suite 409, Lawrence, KS 66044, USA Full list of author information is available at the end of the article with a receiver of a weak signal (say, GPS) even when the separation of their frequency bands exceeds the respective nominal bandwidths of the channels by orders of magnitude. When time domain observations of such far-out-of-band interference are made at the receiver frequency, in a relatively wide bandwidth to avoid excessive broadening of the transients, this interference is likely to appear impulsive. Understanding the mechanism of this interference and its impulsive nature, as initially analyzed in [4], is important for its effective mitigation.

Referring to a signal as impulsive implies that the distribution of the instantaneous power of the signal has a high degree of peakedness relative to some standard distribution, such as the Gaussian distribution. A common quantifier of peakedness would be, for instance, the excess kurtosis [5]. In this article, however, we adopt the measure of peakedness relative to a constant signal as the "excess-to-average power" ratio and use the units 


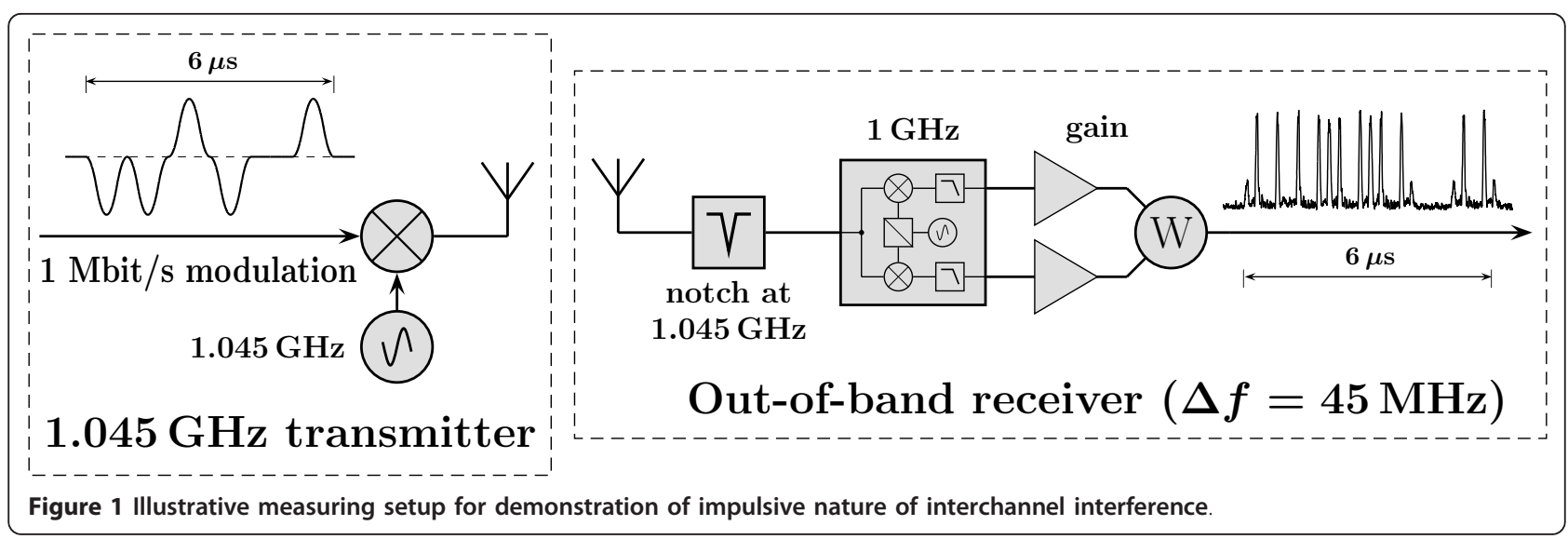

"decibels relative to constant" or $\mathrm{dBc}$. This measure is explained in Appendix A.

Let us consider the illustrative measurement with the setup shown in Figure 1. In the left-hand panel of the figure, the transmitter emits a $1.045-\mathrm{GHz}$ tone with the amplitude modulated by a 'smooth'-looking $1 \mathrm{Mbit} / \mathrm{s}$ message. However, as can be seen in the right-hand panel, the total instantaneous power in an out-of-band quadrature receiver [6] with the bandwidth of several megahertz, tuned to $1 \mathrm{GHz}$, is an impulsive pulse train with a multiple of $250 \mathrm{~ns}$ distance between the pulses.

Figure 2 provides a closer look at the time domain signal traces of the modulating signal (lower panel) and the observed instantaneous power in the receiver (upper panel) for the setup shown in Figure 1. One can see that the power trace is impulsive as its peaks significantly extend above the average power level indicated by the horizontal solid line. Note that some of the peaks of the instantaneous power originate at zero modulation amplitude (at onsets and ends of the modulating pulses), while others originate at the 'smoothest', most linear parts of the modulating signal. The next section clarifies the origins of this impulsive nature of the out-of-band interference.

\section{Impulsive nature of interchannel interference}

As shown in more detail in Appendix B, the signal components induced in a receiver by out-of-band communication transmitters can be impulsive. For example, if the receiver is a quadrature receiver with identical low-pass

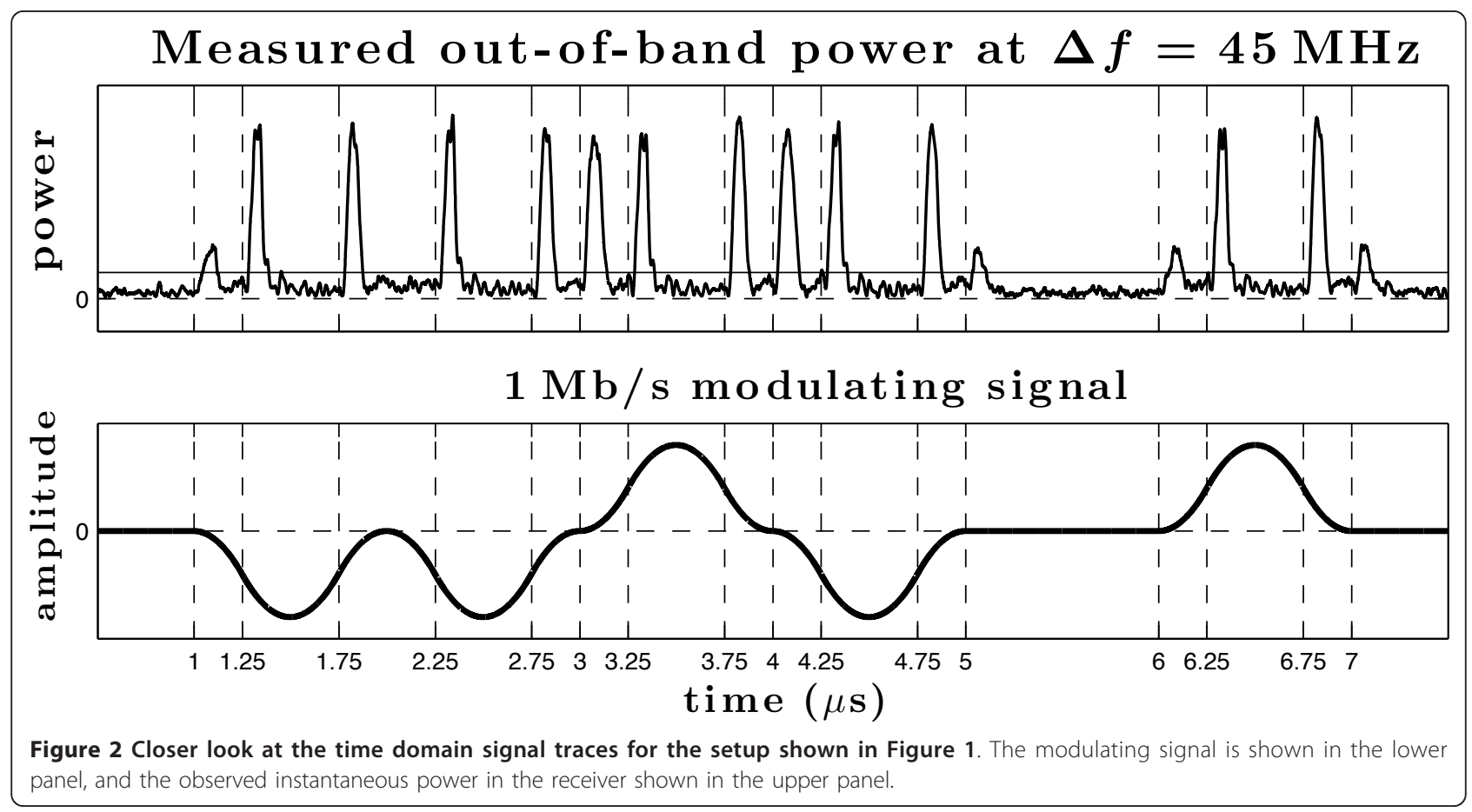


filters in the channels, the main term of the total instantaneous power of in-phase and quadrature components resulting from such out-of-band emissions may appear as a pulse train consisting of a linear combination of pulses originating at discrete times and shaped as the squared impulse response of these filters. For a single transmitter, the typical intervals between those discrete times are multiples of the symbol duration (or other discrete time intervals used in the designed modulation scheme, for example, chip and guard intervals). The non-idealities in hardware implementation of designed modulation schemes such as the non-smooth behavior of the modulator around zero also contribute to additional discrete origins for the pulses. If the typical value of those discrete time intervals is large in comparison with the inverse bandwidth of the receiver, this pulse train may be highly impulsive.

The above paragraph can be restated using mathematical notations as follows. The total emission from various digital transmitters can be written as a linear combination of the terms of the following form:

$$
x(t)=A_{T}(\bar{t}) e^{i \omega_{c} t}
$$

where $\omega_{\mathrm{c}}$ is the frequency of a carrier, $\bar{t}=\frac{2 \pi}{T} t$ is dimensionless time, and $A_{T}(\bar{t})$ is the desired (or designed) complex-valued modulating signal representing a data signal with symbol duration $T$. Let us assume that the impulse response of the low-pass filters in both channels of a quadrature receiver is $w(t)=\frac{2 \pi}{T} h(\bar{t})$ and that the order of the filter is larger than $n$ so that all derivatives of $w(t)$ of order smaller or equal to $n-1$ are

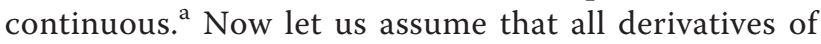
the same order of the modulating signal $A_{T}(\bar{t})$ are finite, but the derivative of order $n-1$ of $A_{T}(\bar{t})$ has a countable number of step discontinuities ${ }^{\mathrm{b}}$ at $\left\{\bar{t}_{i}\right\}$. Then, if $\Delta \omega$ $=2 \pi \Delta f$ is the difference between the carrier and the receiver frequencies, and the bandwidth of the low-pass filter $w(t)$ in the receiver is much smaller than $\Delta f$, the total power in the quadrature receiver due to $x(t)$ can be expressed as ${ }^{\mathrm{c}}$

$$
\begin{aligned}
P_{x}(t, \Delta f)= & \frac{1}{(T \Delta f)^{2 n}} \sum_{i} \alpha_{i} h\left(\bar{t}-\bar{t}_{i}\right) \sum_{j} \alpha_{j}^{*} h\left(\bar{t}-\bar{t}_{i}\right) \\
& \text { for } T \Delta f \gg 1,
\end{aligned}
$$

where $\alpha_{i}$ is the value of the $i$ th discontinuity of the order $n-1$ derivative of $A_{T}(\bar{t})$,

$$
\alpha_{i}=\lim _{\varepsilon \rightarrow 0}\left[A_{T}^{(n-1)}\left(\bar{t}_{i}+\varepsilon\right)-A_{T}^{(n-1)}\left(\bar{t}_{i}-\varepsilon\right)\right] \neq 0 .
$$

A typical value of $t_{i+1}-t_{i}$ would be of the same order of magnitude as $T$. If the reciprocal of this value is small in comparison with the bandwidth of the receiver, the contribution of the terms $\alpha_{i} \alpha_{j}^{*} h\left(\bar{t}-\bar{t}_{i}\right) h\left(\bar{t}-\bar{t}_{j}\right)$ for $i \neq j$ is negligible and (2) describes an impulsive pulse train consisting of a linear combination of pulses shaped as $w^{2}(t)$ and originating at $\left\{t_{i}\right\}$, namely

$$
P_{x}(t, \Delta f)=\frac{1}{(T \Delta f)^{2 n}} \sum_{i}\left|\alpha_{i}\right|^{2} h^{2}\left(\bar{t}-\bar{t}_{i}\right)
$$

for sufficiently large $T$ and $\Delta f$.

Equipped with (4), let us reexamine the time domain traces of the illustrative measurement outlined in Figure 1. In Figure 3, these traces are expanded to include the first two time derivatives of the modulating signal. It can be seen in the figure that (i) the onsets of the power pulses originate at the discontinuities in the second derivative of the modulating signal and (ii) the magnitudes of those pulses are proportional to the squared magnitudes of the discontinuities. Both observations are compliant with (4).

As an additional illustration of a pulse train according to (4), panel I of Figure 4 shows simulated instantaneous total power response of quadrature receivers tuned to 1and 3-GHz frequencies (green and black lines, respectively) to an amplitude-modulated 2-GHz carrier of unit power. The squared impulse response of the low-pass filter in the receiver channels $(30 \mathrm{MHz} 5$ th order Butterworth filter [7] is indicated in the upper right corner of the panel.

The modulating signal is shown in panel II(a) of the figure and represents a random bit sequence at 10 Mbit/s ( $T=100 \mathrm{~ns})$. In this example, a highly oversampled FIR raised cosine filter [6] with roll-off factor 0.35 , and group delay $2 T$ was used for pulse shaping. A rather small group delay was chosen to make the discontinuities in the derivative more visible in the figure. Panel II(b) of Figure 4 shows the first derivative of the modulating signal. This derivative exhibits step discontinuities at the multiple of $T$ time intervals (at the time ticks), and thus $n=2$ in (2).

It is important to notice that the impulsive pulse train is not necessarily caused directly by the discontinuities in the amplitude and/or phase of the transmitted signal, but rather by the discontinuities in the higher order derivatives of the modulating signal, and is generally unrelated to the magnitude of the envelope and/or the peak-to-average ratio of the transmitted signal. Thus, for instance, continuous-phase modulation (CPM), while generally reducing the magnitude of the impulsive inter- 


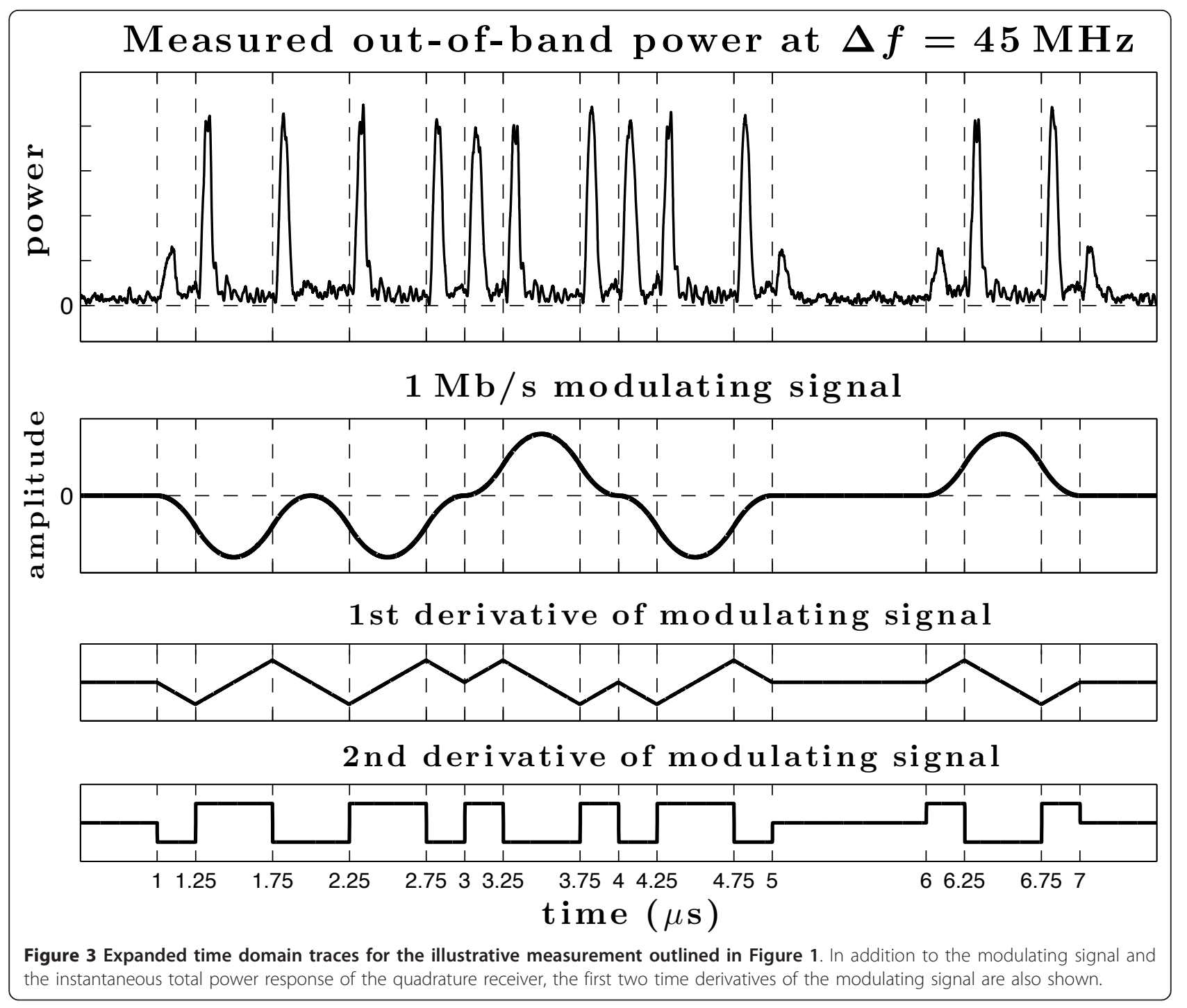

ference by increasing the order of the first discontinuous derivative by one, does not eliminate the effect altogether. This is illustrated in Appendix C.

When viewed as a function of both time and frequency, the interpretation of (2) for the total power in a quadrature receiver is a spectrogram [8] in the time window $w(t)$ of the term $x(t)$ of the transmitted signal. Such a spectrogram is shown in the lower panel of Figure 4, where the horizontal dashed lines indicate the receiver frequencies 1 and $3 \mathrm{GHz}$ used in panel I.

For a quantitative illustration of the impulsive nature of the out-of-band interference, the upper panel of Figure 5 shows the peakedness of the instantaneous total power in a quadrature receiver as a function of frequency for the example used in Figure 4. The peakedness of the out-of-band signal exceeds the peakedness of the in-band signal by over an order of magnitude.
The lower panel of Figure 5 shows, for the same examples, the total excess (solid line) and average (dashed line) power in the receiver versus frequency. The excess power of the out-of-band emissions is approximately $10 \mathrm{~dB}$ higher than the average power.

Given the designed properties of the transmitted signal, the out-of-band emissions can be partially mitigated by additional filtering. For example, one can apply additional high-order low-pass filtering to the modulating signal or band-pass filtering to the modulated carrier. However, the bandwidth of those additional filters must be sufficiently large in comparison with the bandwidth of the pulse shaping filter in the modulator in order to not significantly affect the designed signal. Within that bandwidth, the above analysis still generally holds, and the impulsive disturbances may significantly exceed the thermal noise level in the receiver [3] even when the 


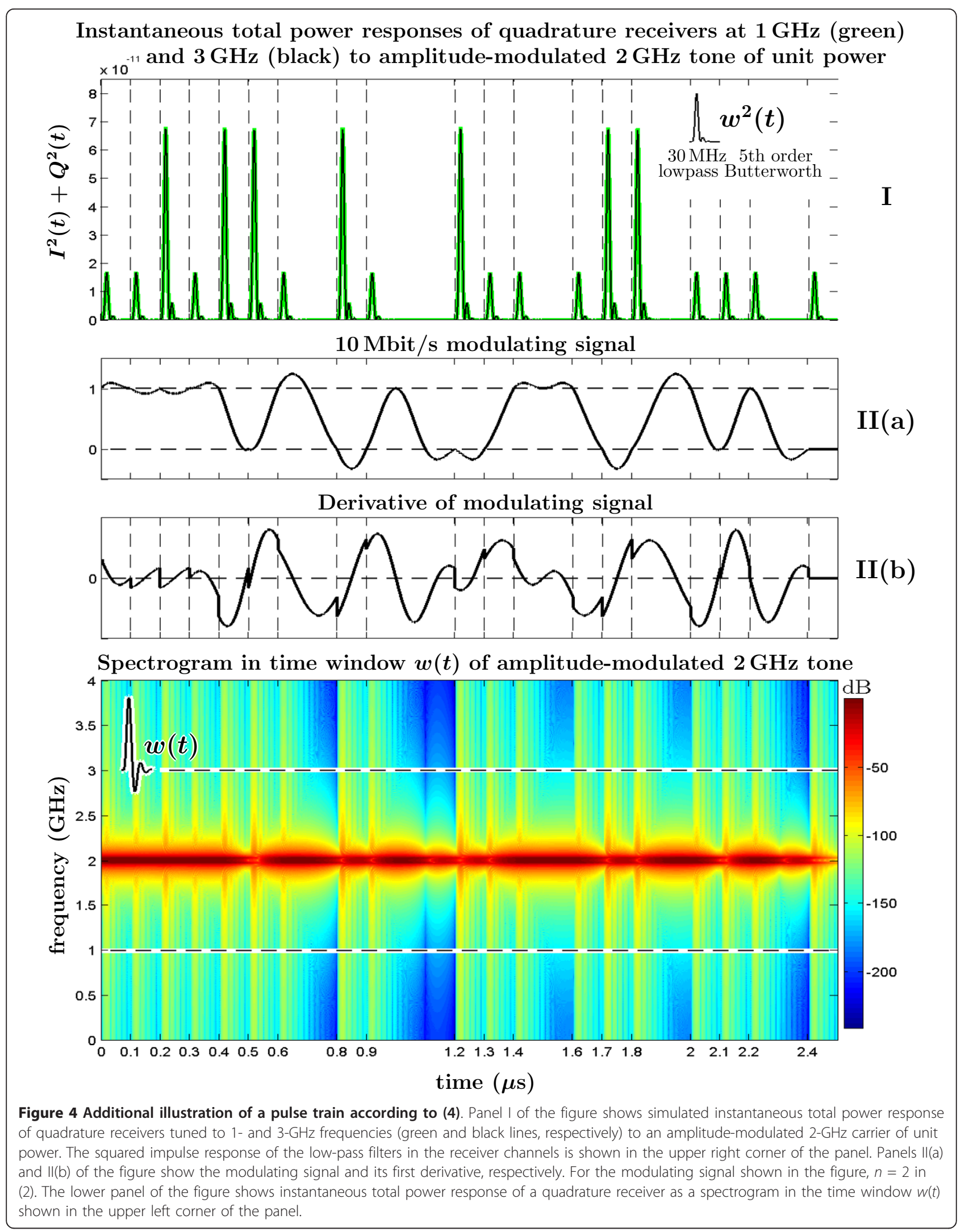




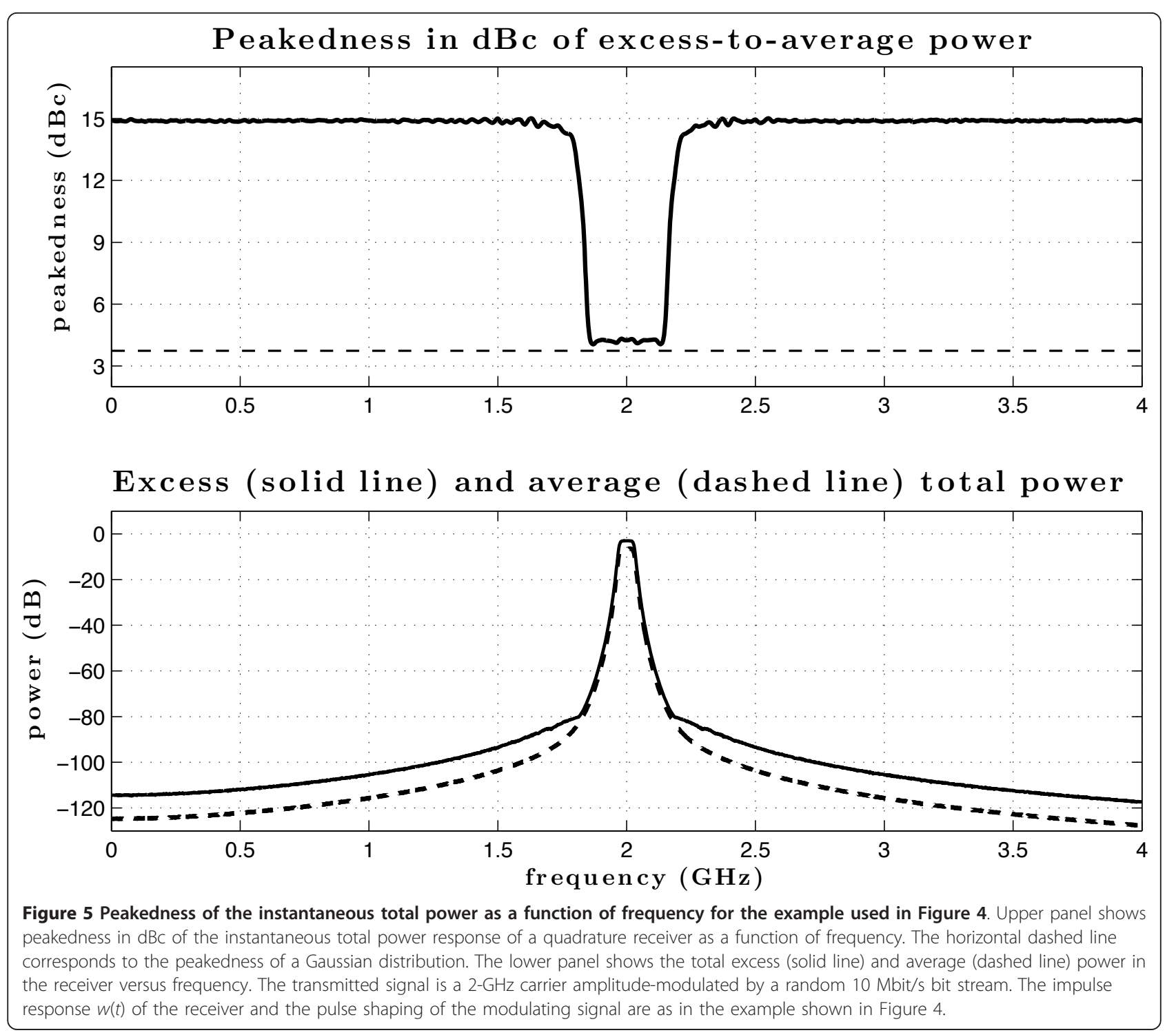

average power of the interference remains below that level.

\section{Conclusions}

Non-smoothness of modulation can be caused by a variety of hardware imperfections and, more fundamentally, by the very nature of any modulation scheme for digital communications. This non-smoothness sets the conditions for the interference in out-of-band receivers to appear impulsive.

If the coexistence of multiple communication devices in, say, a smartphone is designed based on the average power of interchannel interference, a high excess-toaverage power ratio of impulsive disturbances may degrade performance even when operating within the specifications.
On the other hand, the impulsive nature of the interference provides an opportunity to reduce its power. Since the apparent peakedness for a given transmitter depends on the characteristics of the receiver, in particular its bandwidth, an effective approach to mitigating the out-of-band interference can be as follows: (i) allow the initial stage of the receiver to have a relatively large bandwidth so that the transients are not excessively broadened and the out-of-band interference remains highly impulsive, then (ii) implement the final reduction of the bandwidth to within the specifications through nonlinear means, such as the analog filters described in [9-13]. For instance, the differential over-limiter (DoL) described in Appendix D is effective in mitigation of impulsive noise. Using DoL improves the signal-to-noise ratio and increases the data rates of a communication 
channel (e.g., GPS or WCDMA) in the presence of interchannel interference, for example, from WiFi transmissions. An experimental study of the mitigation of the impulsive interference induced in $1.95-\mathrm{GHz}$ High Speed Downlink Packet Access (HSDPA) by 2.4-GHz WiFi transmissions, protocols that coexist in many $3 \mathrm{G}$ smartphones and mobile hotspots, is presented in "Impulsive interference in communication channels and its mitigation by SPART and other nonlinear filters" by AV Nikitin, M Epard, JB Lancaster, RL Lutes, and EA Shumaker, currently under consideration for publication in the EURASIP Journal on Advances in Signal Processing.

\section{Appendix A}

Excess-to-average power ratio as measure of peakedness Consider a signal $x(t)$. Then, the measure $K_{\mathrm{c}}$ of its peakedness in some time interval can be defined implicitly as the excess-to-average power ratio

$$
\left\langle\overline{x^{2}}(t) \theta\left[\overline{x^{2}}(t)-K_{\mathrm{c}}\right]\right\rangle=\frac{1}{2}
$$

where $\theta(x)$ is the Heaviside unit step function, $\langle\ldots\rangle$ denotes averaging over the time interval, and $\overline{x^{2}}(t)=x^{2}(t) /\left\langle x^{2}(t)\right\rangle$ is normalized instantaneous signal power. $K_{\mathrm{c}}=1$ for $x(t)=$ const, and thus $K_{\mathrm{dBc}}=10 \mathrm{lg}$ $\left(K_{\mathrm{c}}\right)$ expresses excess-to-average power ratio in units of "decibels relative to constant".

For a Gaussian distribution, $K_{\mathrm{c}}$ is the solution of

$$
\Gamma\left(\frac{3}{2}, \frac{K_{\mathrm{c}}}{2}\right)=\frac{\sqrt{\pi}}{4}
$$

where $\Gamma(\alpha, x)$ is the (upper) incomplete gamma function [14], and thus $K_{\mathrm{c}} \approx 2.366\left(K_{\mathrm{dBc}} \approx 3.74 \mathrm{dBc}\right)$.

\section{Appendix B}

\section{Derivation of equation (2)}

Let us examine a short-time Fourier transform of a transmitted signal $x(t)$ in a time window $w(t)=\frac{2 \pi}{T} h(\bar{t})$ which vanishes, along with all its derivatives, outside the interval $[0, \infty]$. We will let the window function $w(t)$ represent the impulse response of an analog low-pass filter and be scaled so that $\int_{0}^{\infty} \mathrm{d} t w(t)=1$.

The short-time (windowed) Fourier transform $X(t, \omega)$ of $x(t)$ can be written as

$$
\begin{aligned}
& X(t, \omega)=\int_{-\infty}^{\infty} \mathrm{d} \tau x(\tau) w(t-\tau) \mathrm{e}^{-\mathrm{i} \omega \tau} \\
= & w(t) *\left[x(t) \mathrm{e}^{-\mathrm{i} \omega t}\right] \\
= & w(t) *[x(t) \cos (\omega t)]-\mathrm{i} w(t) *[x(t) \sin (\omega t)] \\
= & I(t, \omega)+\mathrm{i} Q(t, \omega),
\end{aligned}
$$

where the asterisk denotes convolution, and $I(t, \omega)$ and $Q(t, \omega)$ can be interpreted as the in-phase and quadrature components, respectively, of a quadrature receiver with the local oscillator frequency $\omega$ and the impulse response of low-pass filters in the channels $w(t)$.

Let us use the notation for dimensionless time as $\bar{t}=\frac{2 \pi}{T} t$ and consider a transmitted signal $x(t)$ of the form

$$
x(t)=A_{T}(\bar{t}) \mathrm{e}^{\mathrm{i} \omega_{\mathrm{c}} t},
$$

where $\omega_{\mathrm{c}}$ is the frequency of the carrier, and $A_{T}(\bar{t})$ is the desired (or designed) complex-valued modulating signal representing a data signal with symbol duration $T$.

The windowed Fourier transform of $x(t)$ can be written as

$$
\begin{gathered}
X(t, \Delta \omega)=\int_{-\infty}^{\infty} \mathrm{d} \tau A_{T}(\bar{\tau}) w(t-\tau) \mathrm{e}^{\mathrm{i} \Delta \omega \tau} \\
=\frac{2 \pi}{T} \int_{-\infty}^{\infty} \mathrm{d} \tau\left[A_{T}(\bar{\tau}) h(\bar{t}-\bar{\tau})\right]\left[\frac{\mathrm{d}}{\mathrm{d} \tau} \frac{\mathrm{e}^{\mathrm{i} \Delta \omega \tau}}{\mathrm{i} \Delta \omega}\right],
\end{gathered}
$$

where $\bar{\tau}=\frac{2 \pi}{T} \tau$ and $\Delta \omega=2 \pi \Delta f=\omega_{\mathrm{c}}-\omega$. Since $w(t)$ and all its derivatives vanish outside the interval $[0, \infty]$, consecutive integration by parts leads to

$$
\begin{aligned}
& X(t, \Delta f)=\frac{\mathrm{i}^{n}}{(T \Delta f)^{n}} \int_{-\infty}^{\infty} \mathrm{d} \bar{\tau} \mathrm{e}^{\mathrm{i}(T \Delta f) \bar{\tau}} \times \\
& \frac{\mathrm{d}^{n}}{\mathrm{~d} \bar{\tau}^{n}}\left[A_{T}(\bar{\tau}) h(\bar{t}-\bar{\tau})\right]=\frac{\mathrm{i}^{n}}{(T \Delta f)^{n}} \int_{-\infty}^{\infty} \mathrm{d} \bar{\tau} \mathrm{e}^{\mathrm{i}(T \Delta f) \bar{\tau}} \times \\
& \sum_{m=0}^{n}\left(\begin{array}{c}
n \\
m
\end{array}\right) \cdot A_{T}^{(n-m)}(\bar{\tau}) \cdot(-1)^{m} h^{(m)}(\bar{t}-\bar{\tau}),
\end{aligned}
$$

where $\left(\begin{array}{l}n \\ m\end{array}\right)=\frac{n !}{(n-m) ! m !}$ is a binomial coefficient (" $n$ choose $\left.m^{\prime \prime}\right)$.

To analyze the relative contributions of the terms in (10), let us first consider the case where all derivatives of order smaller or equal to $n-1$ of the window function $w(t)$ are continuous, and all derivatives of the same order of the modulating signal $A_{T}(\bar{t})$ are finite, but the derivative of order $n-1$ of $A_{T}(\bar{t})$ has a countable number of step discontinuities at $\left\{\bar{t}_{i}\right\}$ :

$$
\alpha_{i}=\lim _{\varepsilon \rightarrow 0}\left[A_{T}^{(n-1)}\left(\bar{t}_{i}+\varepsilon\right)-A_{T}^{(n-1)}\left(\bar{t}_{i}-\varepsilon\right)\right] \neq 0 .
$$

From (11), it follows that $A_{T}^{(n)}(\bar{t})$ has a piecewise continuous component, as well as a singular component: 


$$
\begin{aligned}
A_{T}^{(n)}(\bar{t})=\sum_{i} \alpha_{i} \delta\left(\bar{t}-\bar{t}_{i}\right) & \\
& +(\text { piecewise continuous function of } \bar{t}),
\end{aligned}
$$

where $\delta(x)$ is the Dirac $\delta$-function [15].

The significance of (12) lies in the sifting (sampling) property of the Dirac $\delta$-function:

$$
\int_{-\infty}^{\infty} \mathrm{d} x \delta\left(x-x_{0}\right) h(x)=h\left(x_{0}\right)
$$

for a continuous $h(x)$. Then, substitution of (12) into (10) leads to the following expression:

$$
\begin{aligned}
X(t, \Delta f)=\frac{\mathrm{i}^{n}}{(T \Delta f)^{n}}\left[\sum_{i} \alpha_{i} h\left(\bar{t}-\bar{t}_{i}\right) \mathrm{e}^{\mathrm{i}(T \Delta f) \overline{\bar{t}}_{i}}\right. \\
\left.\quad+\int_{-\infty}^{\infty} \mathrm{d} \bar{\tau} \mathrm{e}^{\mathrm{i}(T \Delta f) \bar{\tau}} \times(\text { continuous function of } \bar{\tau})\right] .
\end{aligned}
$$

The second term in the square brackets is a Fourier transform of a continuous function, and it becomes negligible in comparison with the first term as the product $T \Delta f$ increases. Thus, for the total power $P(t, \Delta f)$ in a quadrature receiver,

$$
\begin{aligned}
& P_{x}(t, \Delta f)=|X(t, \Delta f)|^{2} \approx \\
& \frac{1}{(T \Delta f)^{2 n}} \sum_{i} \alpha_{i} h\left(\bar{t}-\bar{t}_{i}\right) \sum_{j} \alpha_{j}^{*} h\left(\bar{t}-\bar{t}_{j}\right)
\end{aligned}
$$

for $T \Delta f \gg 1$,

which is Equation (2).

\section{Appendix C}

\section{Discontinuities in continuous-phase modulation}

For continuous-phase modulation (CPM), Equation (1) can be rewritten as

$$
x(t)=A_{T}(\bar{t}) \mathrm{e}^{\mathrm{i} \omega_{c} t}=\left[A_{0} \mathrm{e}^{\mathrm{i}\left(T \Delta f_{c}\right) \int_{-\infty}^{\bar{t}} \mathrm{~d} \tau a_{T}(\tau)}\right] \mathrm{e}^{\mathrm{i} \omega_{c} t},
$$

where $\Delta f_{\mathrm{c}}$ is the frequency deviation. Then, the derivative of $A_{T}(\bar{t})$ is

$$
A_{T}^{\prime}(\bar{t})=\mathrm{i}\left(T \Delta f_{\mathrm{c}}\right) A_{T}(\bar{t}) a_{T}(\bar{t}),
$$

and, if $a_{T}^{(n-2)}(\bar{t})$ contains discontinuities, so does $A_{T}^{(n-1)}(\bar{t})$, and the rest of the analysis of this article holds.

\section{Appendix D}

Differential over-limiter (DoL)

A differential limiter can be defined as the following feedback circuit [13]:

$$
\zeta(\mu, \tau)(t)=\mu \int \mathrm{d} t\left\{\tilde{\mathcal{F}}_{\mu \tau}[z(t)-\zeta(\mu, \tau)(t)]\right\},
$$

where $z(t)$ is the (complex-valued) input signal, $\zeta(\mu, \tau)$ $(t)$ is the output, $\mu$ and $\tau$ are positive rate and time constant parameters, respectively, and $\tilde{\mathcal{F}}_{\alpha}(z)$ is a comparator function with the resolution (linear range) parameter $\alpha$. For a particular differential over-limiter (DoL), the comparator function can be defined as

$$
\tilde{\mathcal{F}}_{\alpha}(z)=\frac{z}{|z|} \tilde{\mathcal{F}}_{\alpha}(|z|)=\frac{z}{|z|}\left\{\begin{array}{l}
\frac{|z|}{\alpha} \text { for }|z|<\alpha \\
\frac{\alpha}{|z|} \text { otherwise }
\end{array} .\right.
$$

When the condition $|z(t)-\zeta(\mu, \tau)(t)|<\mu \tau$ is satisfied, the response of the DoL circuit equals that of an RC integrator with $R C=\tau$. Otherwise, the output has a smaller absolute rate of change than the absolute rate of change of the output of the corresponding $\mathrm{RC}$ integrator. If a DoL circuit with sufficiently small $\tau$ is deployed early in the signal chain of a receiver channel affected by nonGaussian impulsive noise, it can be shown that there exists such rate parameter $\mu$ that maximizes signal-tonoise ratio and improves the quality of the channel. The simplified example shown in Figure 6 illustrates this statement.

In Figure 6, the green lines in all panels show the incoming signal-plus-noise mixture, for both time (separately for the in-phase and the quadrature traces $I / Q$ ) and frequency domains. The incoming signal represents a communication signal with the total bandwidth of 5 $\mathrm{MHz}$, affected by a bandlimited mixture of a thermal (Gaussian) and a white impulsive noises, with the total noise peakedness of $8.8 \mathrm{dBc}$. The signal-to-noise ratio in the baseband is $3 \mathrm{~dB}$, and the bandwidth of the noise is an order of magnitude greater than the channel bandwidth.

The incoming signal is filtered by (i) an RC integrator with the time constant $\tau=16$ ns (black lines in the lefthand panels) and (ii) a DoL circuit with the same $\tau$ and appropriately chosen resolution parameter (black lines in the right-hand panels). Note that the RC integrator is just the DoL circuit in the limit of a large resolution parameter.

As can be seen in the left-hand panels, the RC filter does not affect the baseband signal-to-noise ratio, as it only reduces the power of the noise outside of the channel. Also, since the time constant is small, the noise remains impulsive $(7 \mathrm{dBc})$, as can be seen in the upper panels on the left showing the in-phase/quadrature (I/ Q) time domain traces. On the other hand, the DoL circuit (the right-hand panels) improves the signal-to-noise ratio in the baseband by $7.4 \mathrm{~dB}$, effectively suppressing the impulsive component of the noise and reducing the 


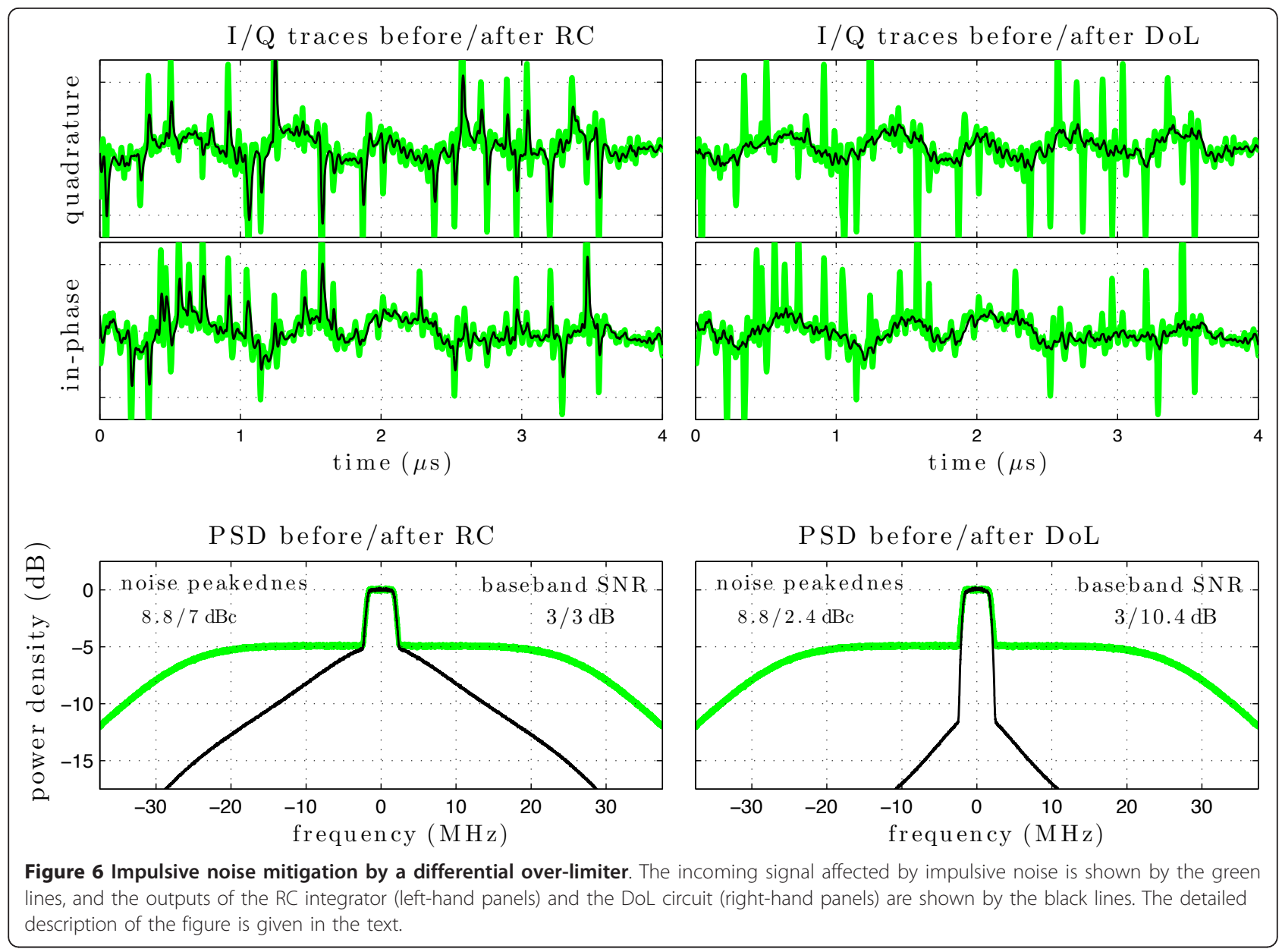

noise peakedness to $2.4 \mathrm{dBc}$. By comparing the black lines in the upper panels of the figure, for the RC and the DoL circuits, one can see how the DoL circuit removes the impulsive noise by "trimming" the outliers while following the narrower-bandwidth trend.

Differential limiters can also be viewed as feedback RC integrators where the time parameter $(\tau=R C)$ is a variable that increases when the absolute value of the difference between the input and the feedback of the output exceeds the resolution parameter $\alpha$. The output $\zeta(\tau, \alpha)$ $(t)$ of such a limiter can be described by

$$
\zeta(\tau, \alpha)(t)=\int \mathrm{d} t \frac{z(t)-\zeta(\tau, \alpha)(t)}{\tilde{\tau}(\tau, \alpha)(|z-\zeta|)},
$$

and the relationship between the variable time parameter $\tilde{\tau}(\tau, \alpha)(|z|)$ and the comparator function $\tilde{\mathcal{F}}_{\alpha}(|z|)$ can be expressed as follows:

$$
\tilde{\tau}(\tau, \alpha)(|z|)=\tau \frac{|z|}{\alpha} \frac{1}{\tilde{\mathcal{F}}_{\alpha}(|z|)} .
$$

Thus, for the DoL circuit, the time parameter is given by

$$
\tilde{\tau}(\tau, \alpha)(|z|)=\left\{\begin{array}{cc}
\tau & \text { for }|z|<\alpha \\
\tau\left(\frac{|z|}{\alpha}\right)^{2} & \text { otherwise }
\end{array} .\right.
$$

A detailed analysis of the DoL filter described by (18) and (19), or by (20) and (22), will be given elsewhere.

\section{Endnotes}

${ }^{a}$ In general, if $n$ is the order of a causal analog filter, then $n-1$ is the order of the first discontinuous derivative of its impulse response. ${ }^{\mathrm{b}}$ One will encounter discontinuities in a derivative of some order in the modulating signal sooner or later, since any physical pulse shaping is implemented using causal filters of finite order. ${ }^{c}$ Equation (2) will still accurately represent the total power in the quadrature receiver if the "real" (physical) modulating signal can be expressed as $A(t)=$ $\psi(t) * A_{T}(t)$, where the convolution kernel $\psi(t)$ is a lowpass filter of bandwidth much larger than $\Delta f$. 


\section{Abbreviations}

CPM: continuous-phase modulation; DoL: differential over-limiter; FIR: finite impulse response; I/Q: in-phase/quadrature; GPS: global positioning system; HSDPA: high speed downlink packet access; WCDMA: wideband code division multiple access; WiFi: wireless fidelity (a branded standard for wirelessly connecting electronic devices).

\section{Acknowledgements}

I express my sincere appreciation to RL Davidchack of the University of Leicester, UK, and to the reviewers of this manuscript for the valuable suggestions and critical comments, and to JB Lancaster of Horizon Analog Inc. (Lawrence KS, USA) for the experimental setup and the data used in Figures 1 through 3.

\section{Author details}

'Avatekh Inc., 900 Masachusetts Street, Suite 409, Lawrence, KS 66044, USA

${ }^{2}$ Horizon Analog Inc., Lawrence, KS, USA

\section{Competing interests}

The author declares that he has no competing interests.

Received: 26 July 2011 Accepted: 21 December 2011

Published: 21 December 2011

\section{References}

1. K Slattery, H Skinner, Platform Interference in Wireless Systems (Elsevier, Amsterdam, 2008)

2. F Leferink, F Silva, J Catrysse, S Batterman, V Beauvois, A Roc'h, Man-made noise in our living environments. Radio Sci Bull. 334, 49-57 (2010)

3. X Yang, A Petropulu, Co-channel interference modeling and analysis in a Poisson field of interferers in wireless communications. IEEE Trans Signal Process. 51, 64-76 (2003). doi:10.1109/TSP.2002.806591

4. AV Nikitin, On the impulsive nature of interchannel interference in digital communication systems, in Proceedings of IEEE Radio and Wireless Symposium, Phoenix, AZ, pp. 118-121 (2011)

5. Abramowitz M, Stegun IA (eds.), Handbook of Mathematical Functions with Formulas, Graphs, and Mathematical Tables, 9th edn. (Dover, New York, 1972)

6. JG Proakis, DG Manolakis, Digital Signal Processing: Principles, Algorithms, and Applications, 4th edn. (Prentice Hall, New Jersey, 2006)

7. R Schaumann, ME Van Valkenburg, Design of Analog Filters (Oxford University Press, Oxford, 2001)

8. L Cohen, Time-Frequency Analysis (Prentice-Hall, Englewood, NJ, 1995)

9. AV Nikitin, RL Davidchack, Signal analysis through analog representation. Proc R Soc Lond A. 459, 1171-1192 (2033)

10. AV Nikitin, RL Davidchack, Adaptive approximation of feedback rank filters for continuous signals. Signal Process. 84(4), 805-811 (2004). doi:10.1016/j. sigpro.2004.01.001

11. AV Nikitin, RL Davidchack, Method and apparatus for analysis of variables. US Patents 7,133,568 (Nov. 7, 2006) and 7,242,808 (July 10, 2007)

12. AV Nikitin, Method and apparatus for real-time signal conditioning, processing, analysis, quantification, comparison, and control. US Patents 7,107,306 (Sep. 12, 2006), 7,418,469 (Aug. 26, 2008), and 7,617,270 (Nov. 10, 2009)

13. AV Nikitin, Method and apparatus for adaptive real-time signal conditioning and analysis. US Patent Application Publication 2011/0112784 (May 12, 2011)

14. IS Gradshteyn, IM Ryzhik, Table of Integrals, Series, and Products, 5th edn. (Academic Press, New York, 1994)

15. PAM Dirac, The Principles of Quantum Mechanics, 4th edn. (Oxford University Press, London, 1958)

doi:10.1186/1687-6180-2011-137

Cite this article as: Nikitin: On the interchannel interference in digital communication systems, its impulsive nature, and its mitigation. EURASIP Journal on Advances in Signal Processing 2011 2011:137.

\section{Submit your manuscript to a SpringerOpen ${ }^{\circ}$ journal and benefit from:}

- Convenient online submission

- Rigorous peer review

- Immediate publication on acceptance

- Open access: articles freely available online

- High visibility within the field

- Retaining the copyright to your article

Submit your next manuscript at $\gg$ springeropen.com 